\title{
Editorial: Advanced intelligent and security services in mobile and ubiquitous computing
}

\author{
Ilsun You • Witold Pedrycz
}

Published online: 18 May 2010

(C) Springer Science+Business Media, LLC 2010

Today research and industry communities are aiming at establishing mobile and ubiquitous computing (MUC) in order to address the emerging needs of the information society. MUC provides the foundation of a "human-oriented communication environment," where people can get online anytime, anywhere, thus having permanent access to their preferred services. With an immense potential to revolutionize our lives, MUC also poses new research challenges.

This special issue concentrates on recent advances and significant contributions to all aspects of the MUC field with an emphasis on forthcoming intelligent and security applications. The issue is composed of six papers, which are significantly extended versions of the original submissions presented at the 3rd International Workshop on Intelligent, Mobile and Internet Services in Ubiquitous Computing (IMIS 2009) held in Fukuoka Institute of Technology, Japan in March 16-19, 2009.

The first article, "A Hybrid Scheduling Scheme for Data Broadcast over Single Channel in Mobile Environments" by Song-Yi Yi et al. introduces a hybrid data broadcast scheme based both on the semantic relationships between data items and the access probabilities. This scheme generates a broadcast schedule, which places semantically related data items together and broadcasts popular data items several times by replicating them in a broadcast cycle. According to the completed simulations, the proposed scheme is efficient with respect to the data access time.

I. You $(\varangle)$

School of Information Science, Korean Bible University,

Seoul, South Korea

e-mail: isyou@bible.ac.kr

\section{W. Pedrycz}

Department of Electrical and Computer Engineering,

University of Alberta, Edmonton, Canada

e-mail: pedrycz@ece.ualberta.ca
The next paper entitled "A Dynamic Service Composition Schema for Pervasive Computing” by Zhuzhong Qian et al. proposes an effective mechanism for automatic service composition based on a two-layered graph in pervasive computing environment. To accomplish this goal, the authors extend the Petri net-based service model and define a novel parameter-based service description to represent services realized in both semantic and syntactic layer. Moreover, a novel service composition scheme is introduced to satisfy user requirements.

In the third paper, "Design and Evaluation of Virtual Home Objects with Music Interaction in Smart Homes” JenqMuh Hsu designs and implements two virtual home objects, Virtual CD and Music Cup, with user-friendly HumanComputer Interaction (HCI) interfaces in order to build an music playback service for a smart home. Home inhabitants can just move these objects through operating gestures upon the implemented HCI interface to activate the virtual home music service. In this way, they can enjoy the intelligent music playback service while reducing the substantial anxiety about the highly technical environment.

The next article, "Efficient Router Nodes Placement for Wireless Mesh Networks" by Fatos Xhafa et al. proposes and evaluates local search methods for intelligent placement of mesh routers in Wireless Mesh Networks (WMNs). The methods explore different local movements and incrementally improve the quality of placement of the nodes of the mesh router. The reported results demonstrate that the proposed methods efficiently achieve their two-fold goal, that is a maximization of the size of the network connectivity and user coverage.

The paper entitled "A Chain-Cluster based Routing Algorithm for Wireless Sensor Networks in Mobile and Ubiquitous Environments" by Feilong Tang et al. introduces a routing algorithm called CCM (Chain-Cluster based Mixed 
routing) for the data gathering applications (e.g., collecting readings concerning amount of water, electricity and gas usages in a building). Especially, the algorithm takes advantages of the two representative algorithms, that is LEACH (Low Energy Adaptive Clustering Hierarchy) and PEGASIS (Power-Efficient Gathering In Sensor Information Systems). It is shown that the proposed algorithm is much better than LEACH and PEGASIS when considering both energy consumption and transmission delay.

Finally, the paper, "Embedded Bandwidth Scalable Wideband Codec Using Hybrid Matching Pursuit Harmonic/CELP Scheme" by Gyu Hyeok Jeong et al. presents a novel hybrid harmocic Code Excited Linear Prediction (CELP) scheme for highband coding of bandsplit scalable wideband codec. In particular, the scheme incorporates a new way of treating speech quality using existing narrowband codecs such as 5.4 and $6.3 \mathrm{kbps}$ G.723.1, 8 kbps G.729, and 11.8 kbps G.729E. In order to evaluate efficient bit allocation and enhanced performance, the pitch of the high-band codec is estimated using the quantized pitch parameter in low-band codec.

We would like to thank the Editor-in-Chief, Prof. Andrew Kusiak, for providing us with the opportunity of bringing forward this Special Issue. We would like to extend special thanks to all authors as well as reviewers for their enthusiasm and dedication, which brought this Special Issue to fruition. 